\title{
Single-Particle Fluorescence Spectrometer for Ambient Aerosols
}

\author{
Yong-Le Pan, ${ }^{1}$ Justin Hartings, ${ }^{2}$ Ronald G. Pinnick, ${ }^{3}$ Steven C. Hill, ${ }^{3}$ \\ Justin Halverson, ${ }^{4}$ and Richard K. Chang ${ }^{1}$ \\ ${ }^{1}$ Department of Applied Physics and Center for Laser Diagnostics, Yale University, New Haven, \\ Connecticut \\ ${ }^{2}$ U.S. Army Medical Research Institute for Infectious Diseases, Ft. Detrick, Maryland \\ ${ }^{3}$ U.S. Army Research Laboratory, Adelphi, Maryland \\ ${ }^{4}$ Westinghouse Savannah River Company, Aiken, South Carolina
}

\begin{abstract}
A fluorescence particle spectrometer (FPS) for real-time measurement of the fluorescence spectra of aerosol particles in the size range 1-10 $\mu \mathrm{m}$ diameter is reported. The prototype FPS has a sufficiently high sample rate (from 5 to $28 \mathrm{l} / \mathrm{min}$ for $3.5 \mu \mathrm{m}$ to $11 \mu \mathrm{m}$ diameter particles) to measure aerosol within buildings at practical rates (from 1 up to 600 particle fluorescence spectra per minute). Previously reported bioaerosol prototype detectors for measurement of single particle spectra (Pan et al., Opt. Lett., 24, 116-118 (1999); Hill et al., Field Anal. Chem. Tech., 3, 221-239 (1999)) were unable to sample the ambient environment; air containing particles had to be forced under pressure into a sample cell. In addition, sample rates were so small (less than $0.01 \mathrm{l} / \mathrm{min}$ ) as to be impractical for most applications. The present design overcomes these deficiencies by the use of an airtight cell that highly concentrates micrometer-sized particles. A virtual impactor first concentrates aerosol particles, which are then drawn under negative pressure through an aerodynamic focusing nozzle in the inlet of the instrument, through the sample region, providing further concentration. The rate of particle spectra measured by the FPS increases significantly when the particle inlet is within a few meters of some common sources of indoor biological particles, e.g., a person coughing, sneezing, or rubbing his skin, or the presence of a dog. The spectra obtained have a variety of spectral shapes. The FPS may be useful in a variety of areas, e.g., in studying and monitoring airborne particles that cause diseases or allergies.
\end{abstract}

Received 11 January 2002; accepted 5 February 2003.

We gratefully acknowledge the financial support from the Department of Energy (through Education R \& D Association of Georgia University, GA0051-1), the U.S. Army Research Laboratory (DAAL01-97-0128), the U.S. Army Research Office (DAAG55-97-1-0349), and the U.S. Air Force Office Scientific Research (F49620-00-1-018202).

Address correspondence to Yong-Le Pan, Department of Applied Physics and Center for Laser Diagnostics, Yale University, New Haven, CT 06520. E-mail: yongle.pan@yale.edu

\section{INTRODUCTION}

Optical techniques are used extensively for aerosol measurement. They are generally nonintrusive, provide essentially realtime data, and are relatively easy to use. Techniques for measuring (1) aerosol scattering using nephelometers (Ray and Garland 1970; Ensor and Waggoner 1970; Heintzenberg and Charlson 1996; Doherty et al. 1999; Gerber et al. 2000), (2) aerosol absorption using photoacoustics (Bruce and Pinnick 1977; Truex and Anderson 1979; Roessler and Faxvog 1979; Arnott et al. 1999), (3) aerosol extinction using transmissometry (Horvath 1993; Smith and Atkinson 2001), and (4) aerosol size and concentration using light scattering particle counters (Hodkinson and Greenfield 1965; Liu et al. 1974; Cooke and Kerker 1975; Knollenberg 1979; Pinnick and Auvermann 1979; Szymanski and Liu 1986; Kerker 1997), have matured significantly over the last 25 years. In addition to advances in hardware (for example, advances in light sources, detectors, and computers), instrument response models for some of these techniques have been developed that put the interpretation of measured data on a sound footing (see, for example, Arnott et al. 1996; Rosen et al. 1997; Pinnick et al. 2000), thereby making the techniques more definitive for aerosol measurement.

Of the techniques mentioned above, particle counters are one of the most widely used. They have been employed for determining estimates of the tropospheric and stratospheric aerosol burden, for monitoring concentrations of particles in clean rooms, and for detecting atmospheric aerosol pollutants. However, these instruments suffer from a critical limitation: they provide almost no information about particle composition. Detecting chemical composition of particles is desirable for a variety of applications, such as in detecting fugitive aerosol emissions, differentiating between biological and nonbiological aerosols, classifying biological particles, and investigating aerosol drug-delivery systems. 
Light scattering particle counters are based on a singleparticle detection approach, wherein particles entrained in an air stream are rapidly drawn through an intense light beam and light scattered by single particles is sensed and used to infer particle size. Recently this approach has been expanded to the measurement of the two-dimensional angular optical scattering of single aerosol particles (Kaye et al. 1997; Holler et al. 1998), laser-induced breakdown spectroscopy of single particles (Hahn and Lunden 2000), laser-ablation mass spectrometry of single particles (Murphy and Thomson 1995; Reilly et al. 1997), and the intrinsic laser-induced fluorescence (LIF) of single particles (Pinnick et al. 1995), all of which may be used for additional characterization.

LIF is particularly useful for detecting biological molecules within aerosol particles (Pinnick et al. 1995; Hairston et al. 1997; Eversole et al. 1999; Reyes et al. 1999; Brosseau et al. 2000; Kaye et al. 2000; Ho 2002). These efforts concentrate on the measurement of the undispersed fluorescence of particles and consequently only have limited potential for providing information on particle composition.

Recently, more capable techniques to measure the LIF spectra of single aerosol particles were developed (Hill et al. 1995; Nachman et al. 1996; Chen et al. 1996; Pinnick et al. 1998; Cheng et al. 1999; Hill et al. 1999) in order to obtain better aerosol classification. In these investigations the emphasis was on detecting biological aerosols using both $\mathrm{cw}$ and pulsed laser sources with wavelengths ranging from $263 \mathrm{~nm}$ to $488 \mathrm{~nm}$. Using a diode-pumped, solid-state, $266 \mathrm{~nm}$ pulsed laser source, a Schwartzchild reflective objective with large numerical aperture for collection of fluorescence, and an intensified-CCD detector mounted on the exit port of a spectrograph, measurement of fluorescence spectra of single bioaerosol particles as small as 2 micrometer diameter was achieved (Pan et al. 1999; Hill et al. 1999).

However, this significant achievement is hampered by critical deficiencies. Their sample cell was not airtight, so aerosol had to be forced under positive air pressure into the sample region. Thus ambient air could not be easily sampled. Further, the requirement for high laser intensity to excite fluorescence (i.e., a tightly focused UV laser beam) and small depth-of-field of the reflective objective (to allow fluorescence light to be collected over a large solid angle and focused onto the spectrograph slit) resulted in a small sample region, which was only on the order of $20 \mu \mathrm{m}$ diameter. The resulting sample rate (for air containing particles to be detected) was less than $0.01 \mathrm{l} / \mathrm{min}$ (Hill et al. 1999), which is impractically low for most applications.

For example, Shaffer and Lighthart (1997) found that culturable bacteria concentrations at urban, rural, forest, and coastal sites ranged from only about 0.06 to 1 colony-forming units per liter. Total bacteria at a rural site were found to have concentrations from about 10 to 100 per liter (Tong and Lighthart 1999). So a sample rate of $0.01 \mathrm{l} / \mathrm{min}$ is far too low for monitoring airborne bacteria in these environments.
These deficiencies have been largely overcome in the development reported here. The sample cell has been made airtight, allowing for sampling of ambient air. Air containing micrometersized particles of interest is drawn first through an aerodynamic virtual impactor concentrator. Aerosol is then drawn from the concentrator through a conically shaped nozzle, where the aerosol particles are aerodynamically focused, providing another stage of aerosol concentration, to intersect the (fluorescenceexciting) laser beam. This combination of virtual impactor and aerodynamic nozzle in the inlet results in the concentration of micrometer-sized particles by a factor of several thousand.

The prototype instrument that embodies this advancement is capable of measuring a variety of spectra in ambient laboratory air with some known sources of particles, such as coughing persons or the presence of a dog. The particulate count rate can be seen to increase when people near the instrument cough, sneeze, or rub their hands, or when a dog enters the room wagging his tail, or when the dog is petted. The spectra recorded are quite variable. We have not yet analyzed the spectra to see what fluorescent molecules are responsible for the spectra, although tryptophan is generally considered to be the contributor for the $330 \mathrm{~nm}$ spectral peak from biological sources. Such an instrument should be useful in a variety of applications, e.g., in monitoring bioaerosols in hazardous work environments, in monitoring the dosage of aerosolized medications, and in monitoring cat allergens, pesticides, or the spread of airborne diseases.

\section{FLUORESCENCE PARTICLE SPECTROMETER (FPS)}

Figure 1 is a block diagram of the FPS. Ambient air is first drawn into a virtual-impactor particle concentrator (Dycor model XMX), in which particles entrained in air sampled at about $770 \mathrm{l} / \mathrm{min}$ are concentrated into an exit flow of about $21 / \mathrm{min}$ (with pressure of about 2 millibar below atmospheric pressure). The manufacturer claims particles in the size range of $1-5 \mu \mathrm{m}$ are concentrated in the exit flow with $30-40 \%$ efficiency. This concentrated particle-laden air is then drawn, under about 4 millibar pressure below atmospheric pressure, through a conically shaped, 18 degree half-angle, $1 \mathrm{~mm}$ diameter nozzle, into an airtight box, and then through an exit port aligned with the nozzle. The nozzle aerodynamically focuses $\mu \mathrm{m}$-sized particles, providing a further concentration enhancement in a focal region about $3 \mathrm{~mm}$ below the nozzle. The focal spot of the particles emanating from the nozzle in this region ranges from about $330 \mu \mathrm{m}$ diameter for $11 \mu \mathrm{m}$ diameter particles, as shown by the image of particles in the inset of Figure 1, to about $700 \mu \mathrm{m}$ for $2.5 \mu \mathrm{m}$ particles. This scattering image was obtained for particles crossing two diode laser beams (described below) and a $532 \mathrm{~nm}$ laser beam sheet, and shows particle trajectories resulting in aerodynamic focusing of the aerosol jet. The focal spot contains the "sample volume" described below, where a pulsed UV laser is directed to excite fluorescence in single particles as they pass through it. 


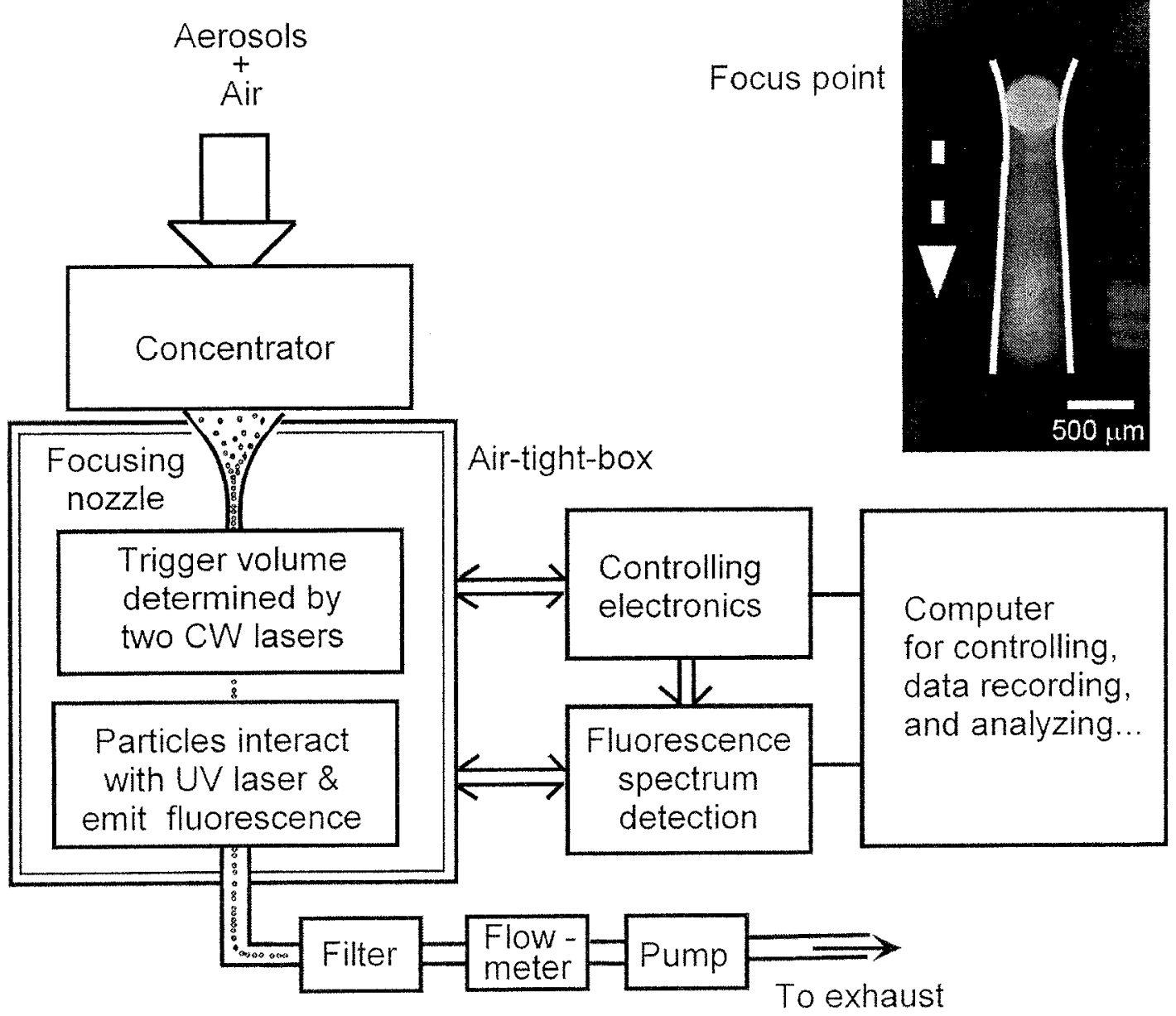

Figure 1. Block diagram of the Fluorescence Particle Spectrometer (FPS). The FPS consists of a virtual impactor/aerodynamic nozzle aerosol concentrator, trigger diode laser subsystem, UV-probe pulsed laser for fluorescence excitation, reflective objective/spectrograph/ICCD detection subsystem for measurement of fluorescence, and computer. The inset (above right) is a timeexposure image of an aerosol particle jet emanating from the focusing inlet nozzle, flowing first through the intersection of the two diode-laser beams that define the "trigger volume" (this region is about $3 \mathrm{~mm}$ below the inlet nozzle, somewhat ellipsoidal in shape, and near the aerosol focal spot), and then (further downstream) through a sheet of light from a $532 \mathrm{~nm}$ laser (forming a cylindrical region). The two curved white lines have been drawn to outline the edge of the particle jet.

The optical arrangement of this system has been described in detail previously (Pan et al. 1999). Briefly, the detection system works as follows. Particles aerodynamically focused within the jet of air from the nozzle are directed toward a trigger volume, defined by the intersection of two (635 and $670 \mathrm{~nm}$ wavelength) focused diode laser beams. When a particle crosses both of these beams a trigger pulse is generated to fire a pulsed UV laser (266 nm Nd:YAG with $0.05 \mathrm{~mJ}$ energy per pulse and pulse length 70 ns; Spectra Physics model Y-70). The laser is set to fire about $1 \mu \mathrm{s}$ after the trigger pulse (the laser cannot fire much before $1 \mu$ s from the trigger, and the electronics pulse shaping and single-channel analyzer circuitry that processes the particle scattering signals from the diode lasers introduces an additional
$1 \mu$ s delay), during which time the particle travels a short distance (about $40 \mu \mathrm{m}$ ) from the trigger volume to the sample volume (a roughly spherical region having diameter about $100 \mu \mathrm{m}$ defined by the intersection of the diode laser beams and the focal region of the collection lens). It is noteworthy that the dispersion in particle velocities caused by different particle accelerations in the nozzle is not a serious issue in the trigger subsystem, since the distance separating the trigger and sample volumes is small.

The UV laser is focused and sufficiently intense (with fluence about 0.03 Joules $/ \mathrm{cm}^{2}$ ) to excite measurable fluorescence in single particles. In addition, the laser spot size is large enough (500 $\mu \mathrm{m}$ spot size) to uniformly illuminate particles within the 
sample volume. The fluorescence from the particles is collected by a Schwartzchild reflecting objective (Ealing) with large numerical aperture (N.A. $=0.5$ ) and focused onto a spectrograph (Acton model SP-150 with 300 groove/mm grating blazed at $500 \mathrm{~nm}$; N.A. $=0.125)$ slit for dispersion. A long-pass filter (N,N-dimethylformamide in a $1 \mathrm{~cm}$ cell) is placed in front of the spectrograph to block the elastic-scattered radiation $(266 \mathrm{~nm}$ wavelength) and to pass the fluorescence. The fluorescence spectra are detected by an image-intensified CCD camera (Princton Instruments) or a 32-anode photo multiplier tube (Hamamatsu model H7260; see Pan et al. 2001) attached to the exit port of the spectrograph.

Air flowing from the nozzle inlet into the airtight cell (an aluminum metal box which contains the trigger diode lasers and detectors, lenses, mounts, translators, and the reflecting objective) is evacuated by a piston pump through an eduction tube, which is aligned with the nozzle and about $2 \mathrm{~cm}$ below it. The pump rate can be adjustable from 0 to $31 / \mathrm{min}$, which determines the sample rate through the nozzle inlet. Measurements reported here were made at $2 \mathrm{l} / \mathrm{min}$ (except as noted below). The exhaust is connected to a filter tube (Baston) before passing through a mass flowmeter (OMEGA Engineering, model 1824 ) and a variable speed piston pump (KNF Neuberger, model UN05).

The overall sample rate of the FPS is dependent on particle size and determined by: (1) the flow rates into the virtual impactor and focusing nozzle, (2) particle losses in the impactor, (3) the concentration enhancement provided by aerodynamic focusing of the inlet nozzle, and (4) the size of the particle jet focal spot compared to the cross-section of the sample volume.

We have measured the sample rate of the FPS using a slight different FPS arrangement than described above. The main differences are: (1) a Spectra Physics model X-30 laser with shorter pulse length (30 ns) was used; (2) pulse delay generators were used in the diode trigger pulse circuitry to increase the length of the diode-beam trigger pulses and thereby increase the particle trigger coincidence rate; (3) a 55 gallon $\left(0.21 \mathrm{~m}^{3}\right)$ air ballast tank with foam insert was placed between the XMX concentrator and the concentrator pump to reduce pressure fluctuations at the outlet of the concentrator induced by the pump; (4) a 2 liter air ballast with foam insert was placed between the air-tight box and the piston pump, also to reduce pressure fluctuation caused by the pump; and (5) the sample rate through the inlet nozzle was reduced to about $0.83 \mathrm{l} / \mathrm{min}$.

The following experimental setup was used for the sample rate measurements. Clean air from a class II biohood (Labconco) was supplied to the virtual impactor concentrator inlet, with precautions taken to minimize changes in the flow rate into the concentrator inlet. An ink jet aerosol generator (IJAG; Bottiger et al. 1998) was also plumbed into the inlet with particle flow directed into the concentrator inlet to minimize particle losses. The IJAG has the capability to generate populations of fairly uniform particles of known size at a known rate, provided that nozzles (the twelve nozzles in the IJAG are fired sequentially) do not plug during the experiment, and provided that the reverse flow in the IJAG drying tube is properly adjusted to effectively sweep out the satellite droplets. For the study here, tryptophan particles, which are strongly fluorescent, were generated at a frequency of about 300/s. Particle size was adjusted by changing the concentration of tryptophan in solution, from a saturated solution of tryptophan in water to $1 / 32$ of this concentration. Corresponding sizes, as measured by collections examined with an optical microscope, have mean diameters ranging from $11 \mu \mathrm{m}$ to $3.5 \mu \mathrm{m}$. Particles generated with the IJAG, not counting agglomerated particles and the small population of particles that derive from satellite droplets that escape the reverse flow in the dryer tube, typically have a $20 \%$ standard deviation in size. For each particle size generated with the IJAG, the rate of trigger events (corresponding to particles with sufficiently large size that passed through both trigger diode laser beams) was measured. Each trigger event resulted in a characteristic tryptophan particle fluorescence spectrum, as measured with the ICCD camera, confirming that the particles were tryptophan. The variability in the amplitude of the spectra (as shown in Figure 2 for nominal $3.5 \mu \mathrm{m}$ diameter particles) is of the order of a factor three, which we attribute to (1) the small depth-of-field of the Schwartzchild objective lens and variable collection efficiency of the lens for out-of-focus particles, (2) nonuniform intensity of the $266 \mathrm{~nm}$ wavelength probe laser beam throughout the sample volume, and (3) nonuniformity of tryptophan particle sizes. Null tests were also made by turning off the IJAG and confirming that the trigger event rate dropped quickly to zero.

The overall sampling rate of the FPS was then determined by measuring the fraction of IJAG particles that resulted in trigger events. Needed for this determination is the Dycor model XMX virtual impactor inlet air flow. Here we have made a measurement of the XMX inlet air flow by measuring the time required to evacuate a collapsible plastic bag with a volume of 440 liters; the sample rate obtained was about $770 \mathrm{1} / \mathrm{min}$. The resulting FPS sample rates are $5,8,18$, and $281 / \mathrm{min}$ for tryptophan particle diameters of 3.4, 5.5, 6.9, and $11 \mu \mathrm{m}$ diameter. According to the manufacturer, the virtual impactor delivers particles 1-5 $\mu \mathrm{m}$ in diameter with 30-40\% efficiency (Edgar 2001). Thus it appears that a substantial increase in the FPS sample rate could be achieved by improving the performance of the aerodynamic focusing nozzle.

\section{RESULTS AND DISCUSION}

The FPS was tested in a room with dimensions $15 \mathrm{~m} \times$ $7 \mathrm{~m} \times 5 \mathrm{~m}$. The room is a research laboratory at Yale University in the basement of a large building that houses offices, classrooms, and other laboratories.

\section{Fluorescence Spectra of Particles in Ambient Air}

Figure 3 shows 200 consecutive single-shot fluorescence spectra taken over a period of about 20 min when no people or 


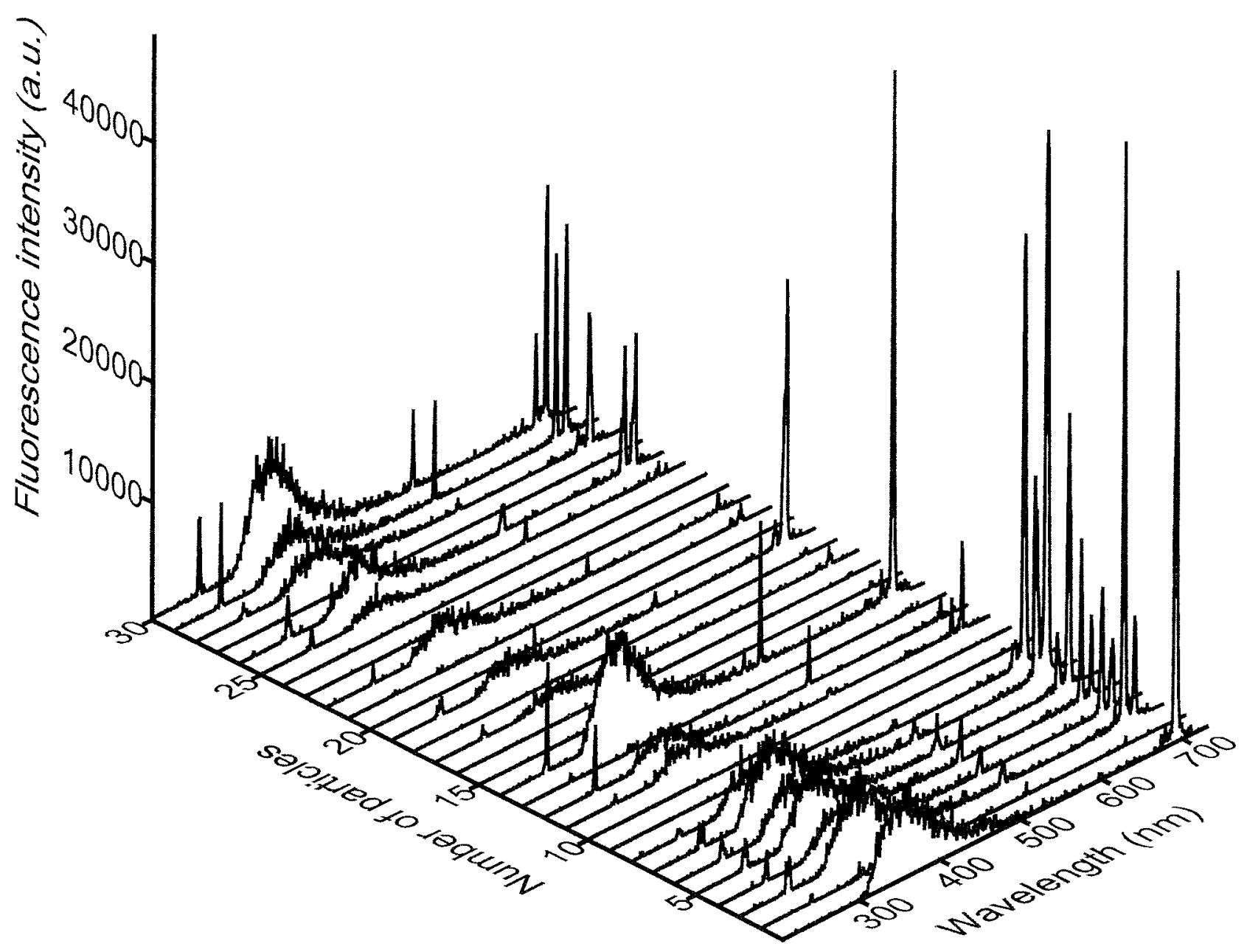

Figure 2. A display of 30 single-shot fluorescence spectra of nominal $3.5 \mu \mathrm{m}$ diameter tryptophan particles measured with the FPS. Variability in the amplitude of the spectra is attributed to (1) the small depth-of-field of the Schwartzchild objective lens and variable collection efficiency of the lens for out-of-focus particles, (2) nonuniform intensity of the $266 \mathrm{~nm}$ wavelength probe laser beam throughout the sample volume, and (3) nonuniform tryptophan particle size.

animals were in the room. The time was late at night, and the particle loading was as low, as we observed. Line-shapes (a), (b), and (c) in the inset indicate typical spectral profiles. Nearly all of the spectral profiles reveal small fluorescence and are flat over the $200 \mathrm{~nm}$ to $650 \mathrm{~nm}$ wavelength range, as in line-shape (d) in the inset. The small sharp peaks at $266 \mathrm{~nm}$ and $532 \mathrm{~nm}$ within some of these spectra are the fourth- and second-harmonic leakages of the Nd:YAG laser. The variability of these peaks is an indication of the variation of aerosol sizes measured. Only a few of these particles fluoresce strongly. As a reference, the fluorescence spectra from four of the primary fluorophors (tyrosine, tryptophan, NADH, and riboflavin) in biological aerosols are shown at the bottom of the inset. Biological aerosols often have a strong peak around $330 \mathrm{~nm}$ resulting from tryptophan (Hill et al. 1999). The variation of the spectra suggests diversity in the composition of the unknown indoor aerosols.
Figure 4 shows the aerosol spectra measured when persons in the room were coughing, scratching, or moving within a couple of meters of the concentrator inlet. The particle count rates were many times larger than when there had been no person in the room. Most of these spectra have a single peak at either $330 \mathrm{~nm}$ or $430 \mathrm{~nm}$, similar to the spectral profiles (a) and (d) shown in the inset. In some of the single-particle spectra, peaks at both $330 \mathrm{~nm}$ and $430 \mathrm{~nm}$ appear with different relative intensities, as in line shapes (b) and (c). The two peaks at $330 \mathrm{~nm}$ and $430 \mathrm{~nm}$ have a width of approximately $60 \mathrm{~nm}$. The $330 \mathrm{~nm}$ peak is similar to the tryptophan peak. Nearly all of the spectra have only these two peaks, but with different relative intensities. A few spectra with flat-topped peaks are caused by the saturation of the detector.

The FPS spectrum capture rate greatly increased when a dog was led into the laboratory to wander and to be petted near the concentrator inlet. Figure 5 shows 200 consecutive 


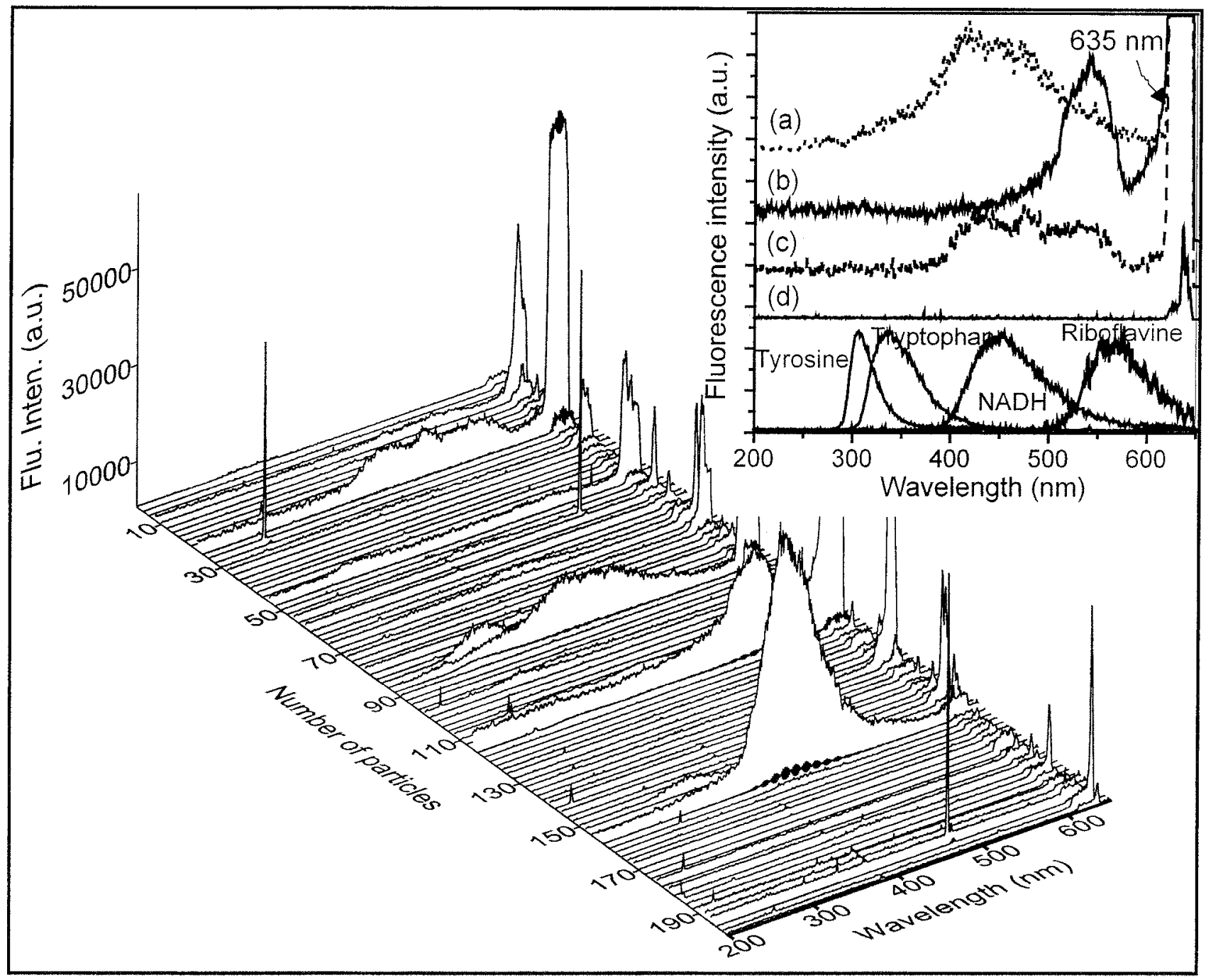

Figure 3. A display of 200 consecutive single-shot fluorescence spectra (266 nm wavelength laser-excitation) of ambient laboratory aerosol measured with the FPS when there was no human activity for about a 20 min period. The spectral peak near $635 \mathrm{~nm}$ is elastic scattering from the trigger diode laser. The inset shows typical spectral lineshapes in (a), (b), (c), and (d). The bottom display (e) in the inset shows the spectra of four of the primary fluorophors in biological aerosols for comparison.

single-shot fluorescence spectra during this period. Typical spectral line-shapes are shown in the inset. Approximately half of the spectra have low fluorescence, similar to that in line shape (d). Line shapes (a), (b), and (c) have two peaks located at $325 \mathrm{~nm}$ and $430 \mathrm{~nm}$ with different relative intensities. These are similar to spectra shown in Figure 4, except that here the peak at $430 \mathrm{~nm}$ is broader (more than $100 \mathrm{~nm}$ at half intensity width).

\section{Response of the FPS to Gas and Diesel Engine Emissions}

In order to investigate the response of the FPS to automobile engine emissions and their possible masking effects for detecting bioaerosols, grab samples of both gasoline engine (a utility van) and a diesel engine (a sedan) emissions were made with 0.2 cubic meter plastic bags. The samples were obtained by connecting empty bags to the exhaust pipes of these vehicles, filling the bags, and quickly transporting them to the laboratory. The fumes in the bags were vented into the FPS virtual impactor inlet within 1 to 2 min of collection. The resulting fluorescence spectra are shown in Figures 6a and 6b. The fluorescence of the exhaust particles from both gasoline and diesel engines are relatively weak, especially from the diesel engine. The fluorescence from the gasoline engine shows two bands peaked at $320 \mathrm{~nm}$ and $410 \mathrm{~nm}$, but with different relative intensities for different 


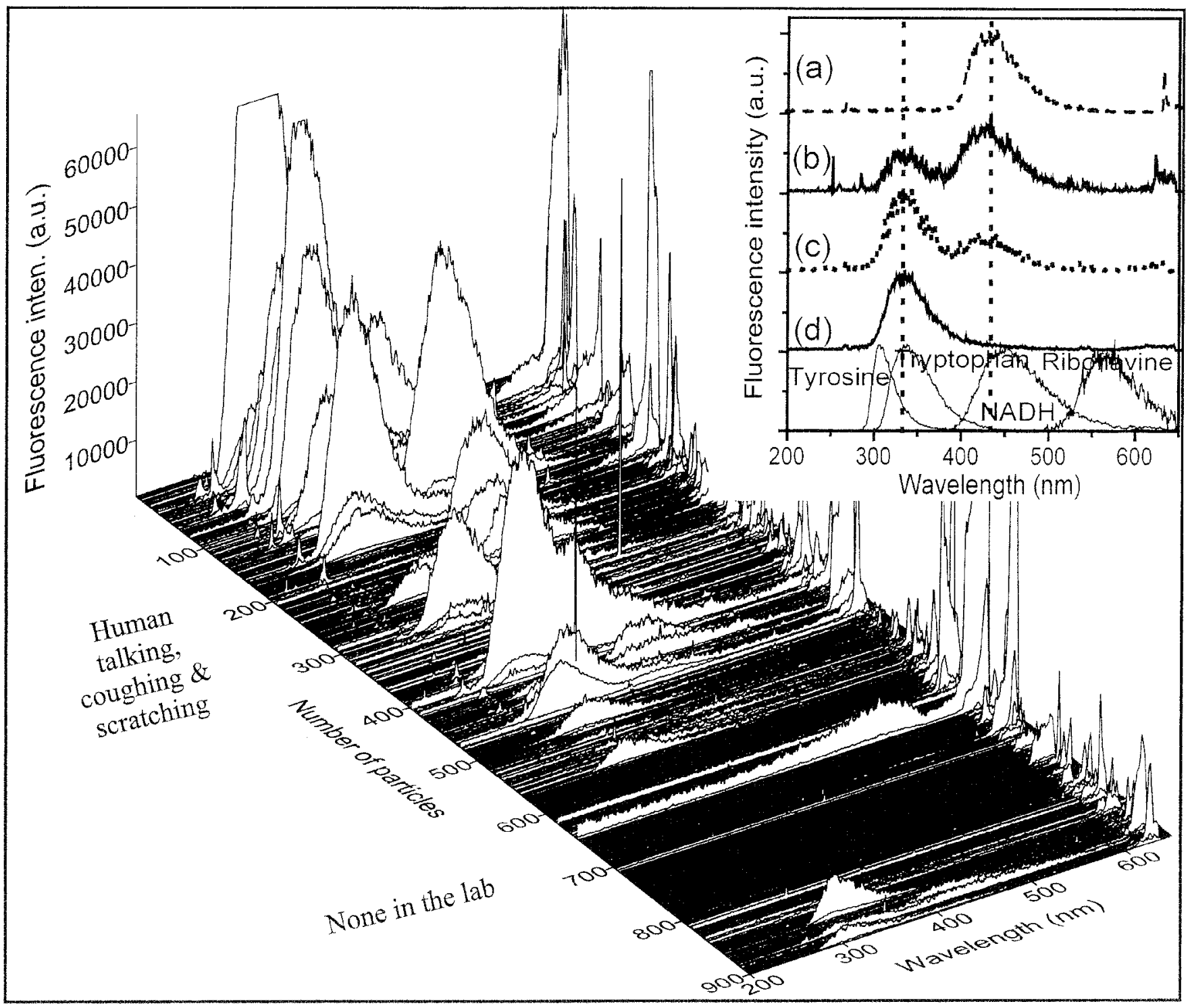

Figure 4. A display of 900 consecutive single-shot fluorescence spectra from ambient laboratory aerosols measured initially while people were talking, coughing, and scratching near the FPS inlet, and then, beginning about trace 550, when no people were in the laboratory. Typical spectral lineshapes are shown in the inset, along with the spectra from four of the primary fluorophors in biological aerosols for comparison.

particles. The fluorescence from the diesel engine shows a single band centered at $400 \mathrm{~nm}$. Our fluorescence observation from single exhaust particles is consistent with the fluorescence spectra obtained from internal combustion engines and stationary burners (Merola et al. 2001). The fluorescence spectrum from diesel and gasoline exhaust generally consists of a UV band around $320 \mathrm{~nm}$ and a visible band around $400 \mathrm{~nm}$, and the spectrum undergoes a red-shift with the increase in the number of aromatic rings and their molecular weight (Merola et al. 2001). Consequently, the fluorescence spectra may be different from the exhausts of different combustion systems with the same fuel or of the same combustion system with different fuels. Figure $6 a$ reveals that the fluorescence spectra from different exhaust particles have different relative intensities of the UV and visible band.

We caution the reader that since typical particle emissions from engines are mostly submicrometer in size, these spectra, which correspond to supermicrometer particles, may not be representative of exhaust particles. We note that since engine emissions have relatively high concentrations of particulates (that 


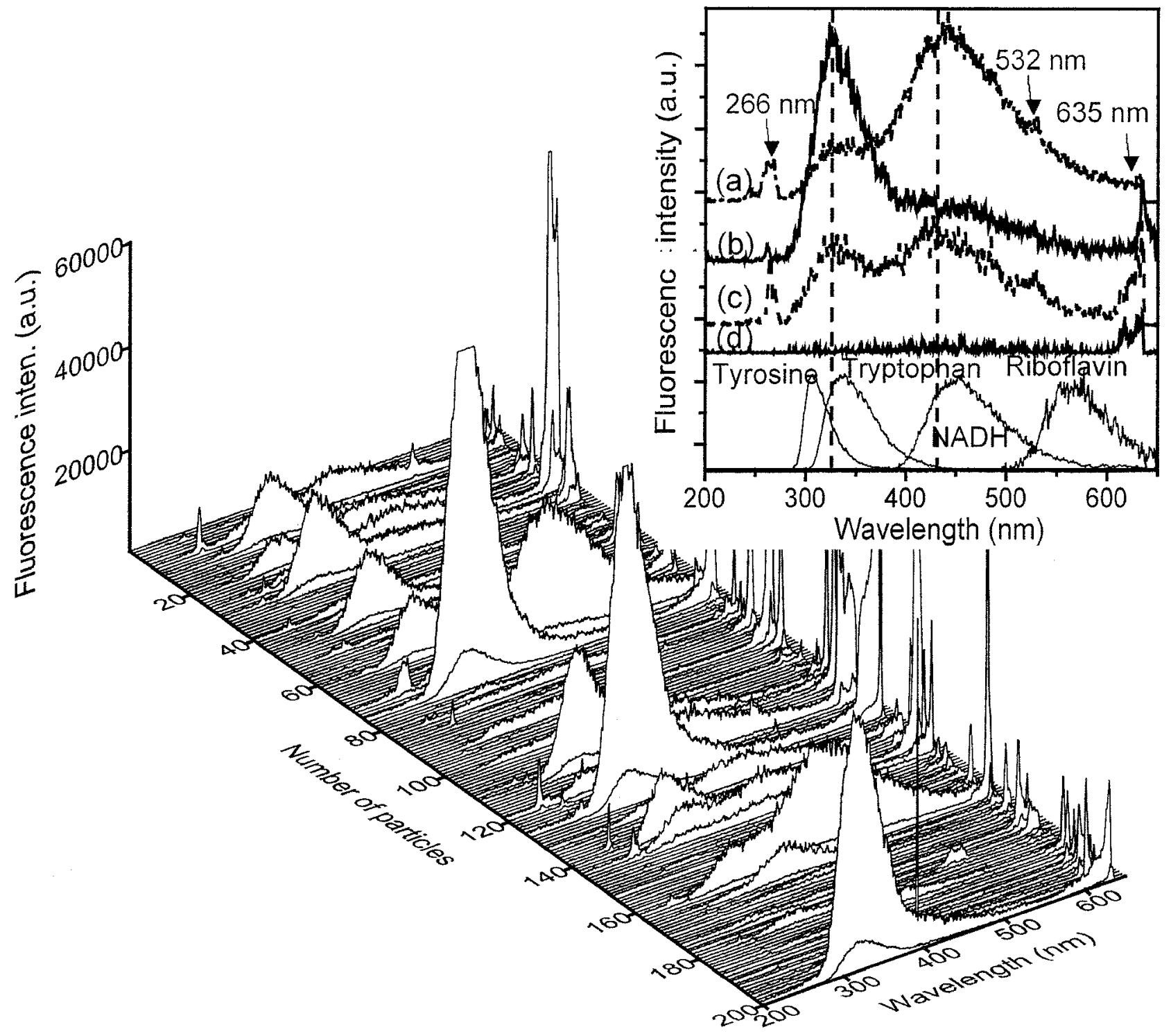

Figure 5. A display of 200 consecutive single-shot fluorescence spectra from ambient laboratory aerosols measured when a dog was walking and being petted near the FPS inlet. Typical spectral lineshapes are shown in the inset, along with the spectra from four of the primary fluorophors in biological aerosols for comparison.

can exceed $10^{6} \mathrm{~cm}^{-3}$ ), some of these spectra may derive from multiple particles in the sample volume.

\section{The FPS Can Measure Spectra at a High Rate}

The FPS was run for $10 \mathrm{~h}$ (from March 2, 16:30 to March 3, $2: 30,2001)$ to monitor the ambient air in the laboratory and to explore the instrument's ability to measure particles at different rates by introducing contaminants. The results are illustrated in Figure 7. During most of the $10 \mathrm{~h}$ period, no one was in the laboratory. However, at the times indicated, powdered materials were released near the concentrator during periods of about $10 \mathrm{~s}$. The center part of Figure 7 shows the histogram of the number of particles (with fluorescence spectra recorded for each particle) captured by the instrument within $10 \mathrm{~min}$ intervals. The histogram reveals the expected large variations in aerosol concentration. In the first $10 \mathrm{~min}$, the instrument measured about 200 particles, a relatively high rate, because the operator was in the room at the beginning of this period. The aerosol concentration gradually decreased until several 10 s of particles were measured every $10 \mathrm{~min}$.

The count rate rapidly increased to 6000 per $10 \mathrm{~min}$ when about $1 \mathrm{mg}$ of tryptophan powder was released near the concentrator inlet at 19:00. Then it fell to several 10s of particles per $10 \mathrm{~min}$ again. The sample rate again increased rapidly during releases of riboflavin and NADH at 20:10 and 22:20. At the times 

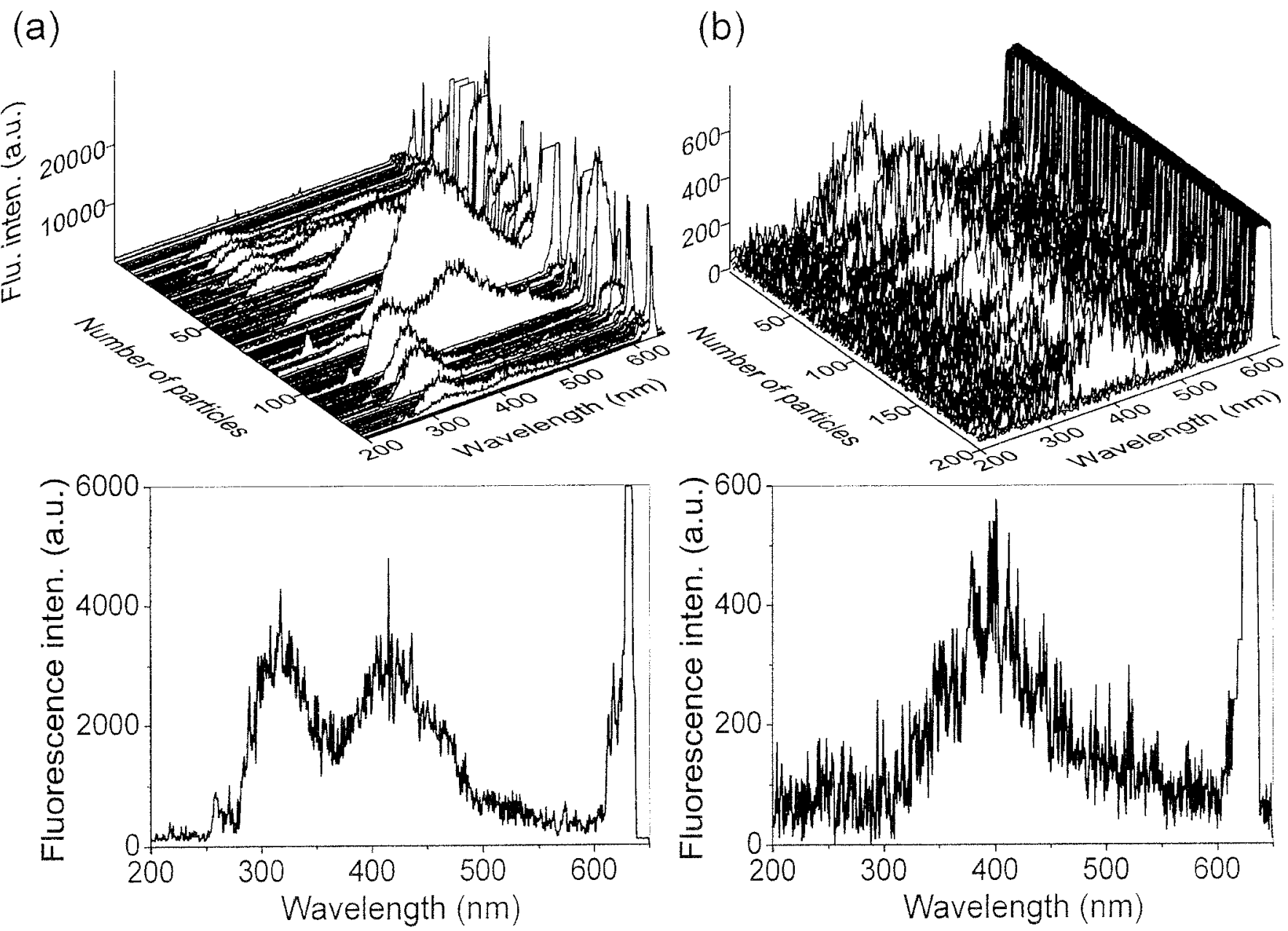

Figure 6. A display of 200 consecutive single-shot FPS fluorescence spectra from grab-samples of gas and diesel engine emissions. Typical spectra are shown for both gas (a) and diesel (b) engine emission particles. Compared to biological particles (Figures 2-4) the fluorescence emissions are weak.

of both releases, the event rate increased to several thousand per 10 min interval. At 21:10, two persons entered the laboratory to check on the instrument, and the sample rate increased slightly. After 22:30, no one was in the room and few people were in the building. The laboratory ambient air became cleaner, and the number of spectra gradually decreased from several 100 to about 10 per $10 \mathrm{~min}$. The small increase during the last $10 \mathrm{~min}$ interval was due to the operator entering the laboratory to turn off the instrument, when a number of spectra were captured in the last few seconds.

Four 1 min segments of this data set are also displayed in Figure 7. In the upper left inset is a typical segment during a quiescent period (without any aerosol release). These spectra have line shapes similar to those in Figure 3. Spectra in the upper right (NADH release), lower left (tryptophan release), and lower right (riboflavin release) are captured with much higher event rate and display the well-known fluorescence peaks at $450 \mathrm{~nm}$, $330 \mathrm{~nm}$, and $560 \mathrm{~nm}$.

\section{SUMMARY AND FUTURE WORK}

A sensor for real-time measurement of aerosol fluorescence spectra has been developed and tested. The FPS can measure UV-excited fluorescence spectra of single ambient aerosol with sample rates of 5 to $28 \mathrm{l} / \mathrm{min}$ for particles ranging from $3.5 \mu \mathrm{m}$ to $11 \mu \mathrm{m}$ diameter. A better method to simultaneously measure aerosol particle size (along with fluorescence spectra) could be done by adding a third diode laser. The FPS is sufficiently sensitive to detect some common sources of indoor biological particles, e.g., a person coughing, sneezing, or rubbing his skin, or the presence of a dog. Fluorescence spectra from different aerosol sources, and from the same source, exhibit a variety of spectral 

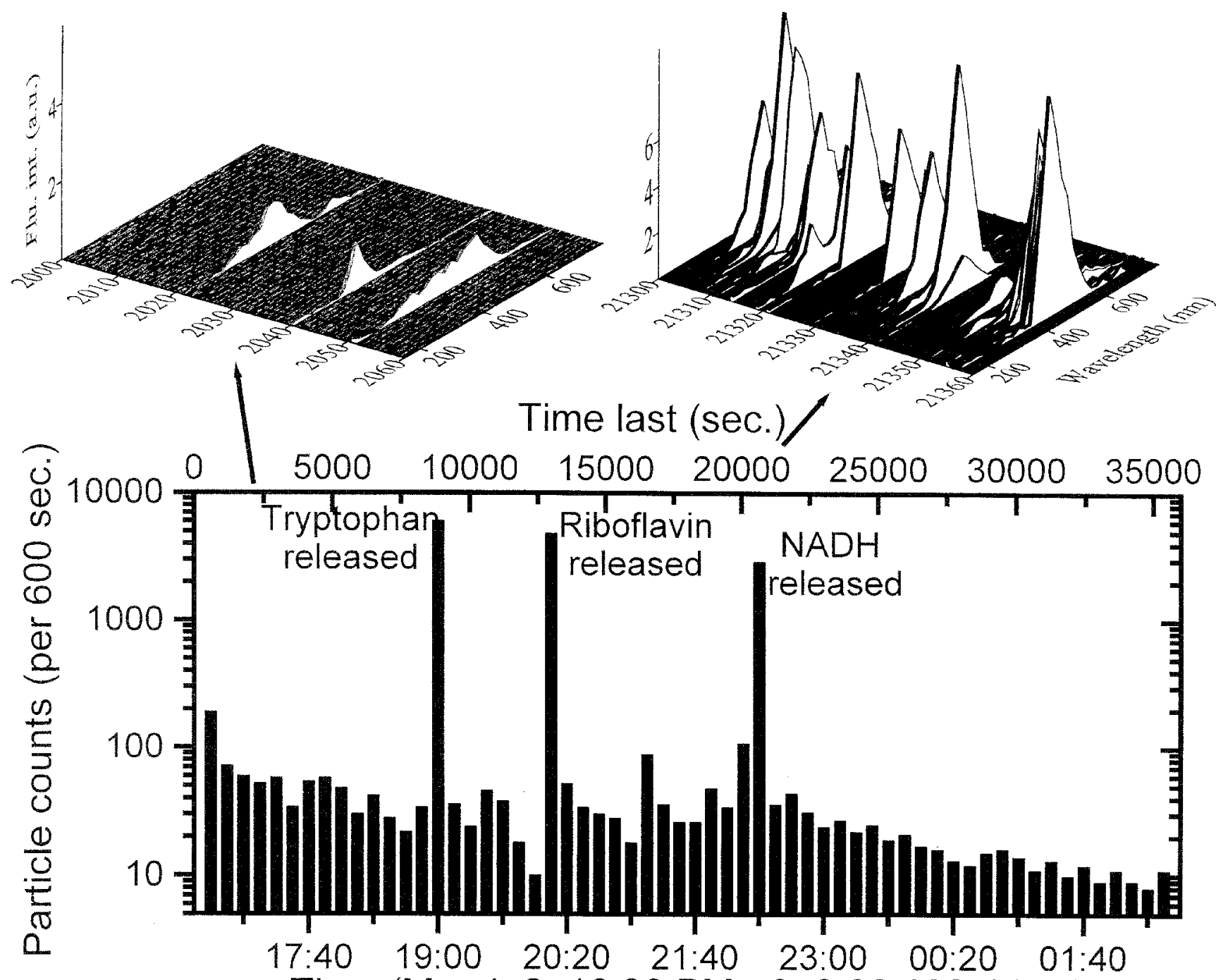

Time (March 2, 16:30 PM - 3, 2:30 AM, 2001)

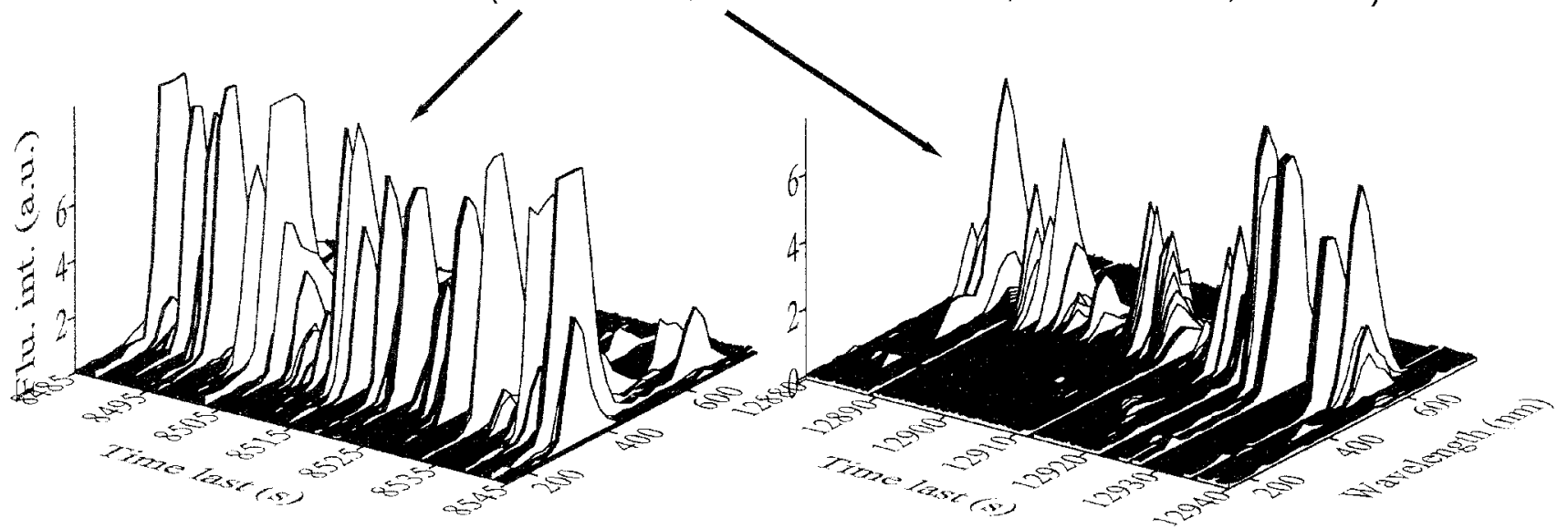

Figure 7. Histogram of the number of FPS fluorescence spectra measured in 10 min intervals (center). At the times indicated, powdered samples of tryptophan, riboflavin, and NADH were released near the FPS inlet for periods of about $10 \mathrm{~s}$. Each of the four three-dimensional displays show the consecutive spectra measured within $1 \mathrm{~min}$ at the times indicated. 
shapes. The FPS may be useful in a variety of applications, e.g., in studying and monitoring airborne particles in workplace environments, and particles that cause allergies, or diseases of animals or plants.

\section{REFERENCES}

Arnott, W. P., Lightfoot, J. A., Raspet, R., and Moosmuller, H. (1996). Radial Wave Thermoacoustic Engines: Theory and Examples for Refrigerators and High-Gain Narrow-Bandwidth Photoacoustic Spectrometers, J. Acoust. Soc. Am. 99:734-745.

Arnott, W. P., Moosmuller, H., Rogers, C. F., Jin, T., and Bruch, R. (1999). Photoacoustic Spectrometer for Measuring Light Absorption by Aerosol: Instrument Description, Atmos. Environ. 33:2845-2852.

Bottiger, J. R., Deluca, P. J., Stuebing, E. W., and Vanreenaen, D. R. (1998). An Ink Jet Aerosol Generator, J. Aerosol Sci. 29(Suppl. 1):S965.

Brosseau, L. M., Vesley, D., Rice, N., Goodell, K., Nellis, M., and Hairston, P. (2000). Differences in Detected Fluorescence Among Several Bacterial Species Measured with a Direct-Reading Particle Sizer and Fluorescence Detector, Aerosol Sci. Technol. 32(6):545-558.

Bruce, C. W., and Pinnick, R. G. (1977). In-Situ Measurements of Aerosol Absorption with a Resonant cw Laser Spectrophone, Appl. Opt. 16:17621765.

Chen, G., Nachman, P., Pinnick, R. G., Hill, S. C., and Chang, R. K. (1996). Conditional-Firing Aerosol-Fluorescence Spectrum Analyzer for Individual Airborne Particles with Pulsed 266-nm Laser Excitation, Opt. Lett. 21:13071309

Cheng, Y. S., Barr, E. B., Fan, B. J., Hargis, P. J., Rader, D. J., O’Hern, T. J., Torczynski, J. R., Tisone, G. C., Preppernau, B. L., Young, S. A., and Radloff, R. J. (1999). Detection of Bioaerosols Using Multiwavelength UV Fluorescence Spectroscopy, Aerosol Sci. Technol. 30(3):186-201.

Cooke, D. D., and Kerker, M. (1975). Response Calculations for Light Scattering Aerosol Counters, Appl. Opt. 14:734-739.

Doherty, S. J., Anderson, T. L., and Charlson, R. J. (1999). Measurement of the Lidar Ratio for Atmospheric Aerosols with a 180 Degree Backscatter Nephelometer, Appl. Opt. 38:1823-1832.

Edgar, G. (2001). Dycor Technologies, Edmonton, Alberta, Canada, private communication.

Ensor, D. S., and Waggoner, A. P. (1970). Angular Truncation Error in the Integrating Nephelometer, Atmos. Environ. 4:481-487.

Eversole, J. D., Hardgrove, J. J., Cary, Jr., W. K., Choulas, D. P., and Seaver, M. (1999). Continuous, Rapid Biological Aerosol Detection with the Use of UV Fluorescence: Outdoor Test Results, Field Anal. Chem. Tech. 3(4-5):249259

Gerber, H., Takano, Y., Garrett, T. J., and Hobbs, P. (2000). Nephelometer Measurements of the Asymmetry Parameter, Volume Extinction Coefficient, and Backscatter Ratio in Arctic Clouds, J. Atmos. Sci. 57:3021-3034.

Hahn, D. W., and Lunden, M. M. (2000). Detection and Analysis of Aerosol Particles by Laser-Induced Breakdown Spectroscopy, Aerosol Sci. Tech. 33:3048.

Hairston, P. P., Ho, J., and Quant, F. R. (1997). Design of an Instrument for Real-Time Detection of Bioaerosols Using Simultaneous Measurement of Particle Aerodynamic Size and Intrinsic Fluorescence, Aerosol. Sci. Tech. 28:471-482.

Heintzenberg, J., and Charlson, R. J. (1996). Design and Application of the Integrating Nephelometer: A Review, J. Atmos. Oceanic Technol. 13:9871000 .

Hill, S. C., Pinnick, R. G., Nachman, P., Chen, G., Chang, R. K., Mayo, M. W., and Fernandez, G. L. (1995). Aerosol-Fluorescence Spectrum Analyzer: RealTime Measurement of Emission Spectra of Individual Airborne Particles, Appl. Opt. 33:7149-7155.

Hill, S. C., Pinnick, R. G., Niles, S., Pan, Y. L., Holler, S., Chang, R. K., Bottiger, J., Chen, B. T., Orr, C. S., and Feather, G. (1999). Real-Time Measurement of
Fluorescence Spectra from Single Airbore Biological Particles, Field Anal. Chem. Tech. 3(4-5):221-239.

Ho, J. (2002). Review: Future of Biological Aerosol Detection, Analytica Chimica Acta 457:125-148.

Hodkinson, J. R., and Greenfield, J. R. (1965). Response Calculations for LightScattering Aerosol Counters, Appl. Opt. 4:1463-1474.

Holler, S., Pan, Y., Chang, R. K., Bottiger, J. R., Hill, S. C., and Hillis, D. B. (1998). Two-Dimensional Angular Optical Scattering for the Characterization of Airborne Microparticles, Opt. Lett. 23:1489-1491.

Horvath, H. (1993). Comparison of Measurements of Aerosol Optical Absorption by Filter Collection and Transmissometric Method, Atmos. Environ. 27:319-325.

Kaye, P. H., Barton, J. E., Hirst, E., and Wang-Thomas, Z. (1997). NeuralNetwork-Based Spatial Light-Scattering Instrument for Hazardous Airborne Fiber Detection, Appl. Opt. 36:6149-6156.

Kaye, P. H., Barton, J. E., Hirst, E., and Clark, J. M. (2000). Simultaneous Light Scattering and Intrinsic Fluorescence Measurement for the Classification of Airborne Particles, Appl. Opt. 39:3738-3745.

Kerker, M. (1997). Light Scattering Instrumentation for Aerosol Studies: An Historical Overview, Aerosol Sci. Tech. 27(4):522-540.

Knollenberg, R. G. (1979). Single Particle Light Scattering Spectrometers. In Aerosol Measurement, edited by D. A. Lundgren, F. S. Harris, W. H. Marlow, M. Lippman, W. E. Clark, and M. Durham. University Press of Florida, Florida, pp. 271-293.

Liu, B. Y. H., Berglund, R. N., and Agarwal, J. K. (1974). Experimental Studies of Optical Particle Counters, Atmos. Environ. 8:717-732.

Merola, S. S., Gambi, G., Allouis, C., Beretta, F., Borghese, A., and D’Alessio, A. (2001). Analysis of Exhausts Emitted by Internal Combustion Engines and Stationary Burners, by Means of UV Extinction and Fluorescence Spectroscopy, Chemosphere 42:827-834.

Murphy, D. M., and Thomson, D. S. (1995). Laser Ionization Mass Spectrometry of Single Aerosol Particles, Aerosol Sci. Tech. 22:237-249.

Nachman, P., Chen, G., Pinnick, R. G., Hill, S. C., Chang, R. K., Mayo, M. W., and Fernandez, G. L. (1996). Conditional-Sampling Spectrograph Detection System for Fluorescence Measurements of Individual Airborne Biological Particles, Appl. Opt. 35:1069-1076.

Pan, Y. L., Cobler, P., Rhodes, S., Potter, A., Chou, T., Holler, S., Chang, R. K., Pinnick, R. G., and Wolf, J.-P. (2001). High-speed High-sensitivity Aerosol Fluorescence Spectrum Detection Using a 32-Anode Photomultiplier Tube Detector, Rev. Sci. Instr. 72:1831-1836.

Pan, Y. L., Holler, S., Chang, R. K., Hill, S. C., Pinnick, R. G., Niles, S., and Bottiger, J. R. (1999). Single-Shot Fluorescence Spectra of Individual Microsized Bioaerosols Illuminated by a $351 \mathrm{~nm}$ or $266 \mathrm{~nm}$ Laser, Opt. Lett. 24(2):116-119.

Pinnick, R. G., and Auvermann, H. J. (1979). Response Characteristics of Knollenberg Light-Scattering Aaerosol Counters, J. Aerosol Sci. 10:55-74.

Pinnick, R. G., Hill, S. C., Nachman, P., Pendleton, J. D., Fernandez, G. L., Mayo, M. W., and Bruno, J. G. (1995). Fluorescnce Particle Counter for Detecting Airborne Bacteria and Other Biological Particles, Aerosol. Sci. Tech. 23:653664.

Pinnick, R. G., Hill, S. C., Nachman, P., Videen, G., Chen, G., and Chang, R. K. (1998). Aerosol Fluorescence Analyzer for Rapid Measurement of Single Micrometer-Sized Airborne Biological Particles, Aerosol Sci. Tech 28:95-104.

Pinnick, R. G., Pendleton, D. J., and Videen, G. (2000). Response Characteristics of the Particle Measuring Systems Active Scattering Aerosol Spectrometer Probes, Aerosol Sci. Technol. 33:334-352.

Ray, J. B., and Garland, J. A. (1970). An Integrating Nephelometer for Atmospheric Studies and Visibility Warning Devices, Atmos. Environ. 4:219-223.

Reyes, F. L., Jeys, T. H., Newbury, N. R., Primmerman, C. A., Rowe, G. S., and Scanchez, A. (1999). Bio-Aerosol Fluorescence Sensor, Field Anal. Chem. Tech. 3(4-5):240-248.

Reilly, P. T. A., Gieray, R. A., Yang, M., Whitten, W. G., and Ramsey, J. M. (1997). Tandem Mass Spectrometry of Individual Airborne Microparticles, Anal. Chem. 69:36-39. 
Roessler, D. M., and Faxvog, F. R. (1979). Optoacoustic Measurement of Optical Absorption in Acetylene Smoke, J. Opt. Soc. Am. 69:1699-1704.

Rosen, J. M., Pinnick, R. G., and Garvey, D. M. (1997). Nephelometer Optical Response Model for the Interpretation of Atmospheric Aerosol Measurements, Appl. Opt. 36:2642-2649.

Smith, J. D., and Atkinson, D. B. (2001). A Portable Pulsed Cavity Ring-Down Transmissometer for Measurement of the Optical Extinction of the Atmospheric Aerosol, Analyst 126:1216-1220.

Szymanski, W. W., and Liu, B. Y. H. (1986). On the Sizing Accuracy of Laser Optical Particle Counters, Part. Charact. 3:1-7.
Shaffer, B. T., and Lighthart, B. (1997). Survey of Culturable Airborne Bacteria at Four Diverse Locations in Oregon: Urban, Rural, Forest, and Coastal, Microbial Ecology. 34:167-177.

Schuster, B. G., and Knollenberg, R. (1972). Detection and Sizing of Small Particles in an Open Cavity Gas Laser, Appl. Opt. 11:1515-1520.

Tong, Y., and Lighthart, B. (1999). Diurnal Distribution of Total Culturable Atmospheric Bacteria at a Rural Site, Aerosol Sci. Technol. 30:246254.

Truex, T. J., and Anderson, J. E. (1979). Mass Monitoring of Carbonaceous Aerosols with a Spectrophone, Atmos. Environ. 13:507-509. 